\title{
Surface waves in a cylindrical borehole through partially-saturated porous solid
}

\author{
M D SHARMa \\ Department of Mathematics, Kurukshetra University, Kurukshetra 136 119, India. \\ e-mail: mohan_here@rediffmail.com
}

MS received 20 September 2016; revised 29 March 2017; accepted 10 June 2017; published online 14 February 2018

Propagation of surface waves is discussed in a cylindrical borehole through a liquid-saturated porous solid of infinite extent. The porous medium is assumed to be a continuum consisting of a solid skeletal with connected void space occupied by a mixture of two immiscible inviscid fluids. This model also represents the partial saturation when liquid fills only a part of the pore space and gas bubbles span the remaining void space. In this isotropic medium, potential functions identify the existence of three dilatational waves coupled with a shear wave. For propagation of plane harmonic waves along the axially-symmetric borehole, these potentials decay into the porous medium. Boundary conditions are chosen to disallow the discharge of liquid into the borehole through its impervious porous walls. A dispersion equation is derived for the propagation of surface waves along the curved walls of no-liquid (all gas) borehole. A numerical example is studied to explore the existence of cylindrical waves in a particular model of the porous sandstone. True surface waves do not propagate along the walls of borehole when the supporting medium is partially saturated. Such waves propagate only beyond a certain frequency when the medium is fully-saturated porous or an elastic one. Dispersion in the velocity of pseudo surface waves is analysed through the changes in consolidation, saturation degree, capillary pressure or porosity.

Keywords. Cylindrical waves; phase velocity; dispersion; porous solid; partial saturation; multiphase pore-fluid.

\section{Nomenclature}

$t \quad$ Time

$\omega \quad$ Angular frequency

$c \quad$ Apparent phase velocity

$k \quad$ Wave number

$f \quad$ Total porosity

$\sigma \quad$ Fraction of pore space filled with liquid

$\delta_{g} \quad$ Volume fraction of gas-filled pores in porous aggregate

$\delta_{l} \quad$ Volume fraction of liquid-filled pores in porous aggregate

$\delta_{s} \quad$ Volume fraction of solid grains in porous aggregate $\rho_{s}, \rho_{g}, \rho_{l}$

$K_{s}, K_{g}, K_{l}$

$G_{s}$

$G_{p}$

$K_{p}$

$K_{c}$

$a$

$\alpha_{j}(j=1,2,3)$

$\beta$
Densities of solid grains, pore-gas and pore-liquid, respectively

Bulk moduli of solid grains, pore-gas, pore-liquid, respectively

Shear modulus of solid grains

Shear modulus of porous frame

Bulk modulus of porous frame

Bulk modulus equivalent of capillary pressure

Radius of cylindrical borehole

Velocities of $P_{1}, P_{2}, P_{3}$ waves, respectively

Velocity of shear wave 


$\begin{array}{ll}(r, \theta, z) & \text { Cylindrical coordinate system } \\ \mathbf{u}=\left(u_{r}, u_{\theta}, u_{z}\right) & \text { Displacement of solid particles } \\ \mathbf{v}=\left(v_{r}, v_{\theta}, v_{z}\right) & \begin{array}{l}\text { Displacement of pore-gas parti- } \\ \text { cles }\end{array} \\ \mathbf{w}=\left(w_{r}, w_{\theta}, w_{z}\right) & \begin{array}{l}\text { Displacement of pore-liquid par- } \\ \text { ticles }\end{array} \\ \tau_{i j}^{(p)} & \text { Stress tensor for porous frame } \\ p^{(g)} & \text { Internal pressure of pore-gas } \\ p^{(l)} & \text { Internal pressure of pore-liquid } \\ a_{i j} & \text { Elastic coefficients } \\ \delta_{i j} & \text { Kronecker symbol } \\ \chi & \text { Capillary parameter for liquid- } \\ & \text { gas saturation } \\ c_{K} & \text { Consolidation parameter for } \\ & \text { incompressibility } \\ c_{G} & \text { Consolidation parameter for } \\ \xi & \text { shear } \\ \xi & \text { Non-dimensional frequency } \\ & \text { parameter }\end{array}$

\section{Introduction}

The propagation of seismic waves in saturated porous media and related phenomena are of great interest in various fields, viz., acoustics, biomechanics, structural engineering, seismology and exploration of subsurface resources. Pores and fractures are pervasive in almost all the rocks in the earth's crust. In-situ reservoir rocks contain oil, water or gas, which are of great economic importance. But, any measurement below the Earth's surface requires drilling of a hole. Then, any study for seismic exploration is concerned with the propagation of seismic waves in and around the cylindrical boreholes (Biot 1952). Cheng et al. (1987) studied the effects of in-situ permeability on the propagation of Stoneley waves in a borehole. Sharma and Gogna (1990) studied elastic wave propagation in a cylindrical borehole through a poroelastic solid of infinite extent. Chao et al. (2004) used shock tube technique to measure the phase velocity of pseudo-Stoneley wave along boreholes in a water-saturated porous reservoir. An agreement with the computations, based on Biot's theory of poroelasticity (Biot 1956), was observed. In a later study, Chao et al. (2007) solved Biot equations in spherical coordinates to analyse the influence of gas bubbles on the propagation of Stoneley wave along a borehole in saturated poroelastic formation. Frequency-dependent bulk modulus of the gas-brine mixture was obtained as the harmonic mean of frequency-dependent bulk modulus of the bubble and the bulk modulus of brine.

The dynamical equations formulated by Biot (1956, 1962a,b) are, generally, used to derive mathematical models for wave propagation studies in liquid-saturated porous solids. However, it is very likely that pores in any geological medium may not be saturated completely with a single-phase fluid. Then, a poroelastic solid saturated with twin-fluid mixture represents a fairly realistic model for sedimentary or reservoir rocks (Brutsaert 1964; Bowen 1976). For convenience, the two fluid phases in pores are identified as liquid and gas. Then, partial or patchy saturation is obtained as a special case when liquid fills only a part of the pore space and a rarefied gas occupies the remaining pore space. Garg and Nayfeh (1986) studied the propagation of compressional waves in porous materials saturated with chemically non-reactive miscible liquid/gas mixture. Santos et al. (1990a,b) derived governing equations and presented a method to calculate elastic constants for isotropic porous solids saturated by two-phase fluids. Comprehensive procedures relevant to wave propagation in porous solids saturated with multiphase fluids are also found in Corapcioglu and Tuncay (1996) and Tuncay and Corapcioglu (1997). Mathematical model presented by Lo et al. (2005) is also based on continuum mixture theory. This theory is general enough to account for the changes in capillary pressure and viscous/inertial coupling among constituents. Using the theory of mixtures, Li and Schanz (2011) established a dynamic three-phase model and used it to obtain the analytical solution for wave propagation in a 1-D partially saturated poroelastic column. Very recently, the author (Sharma 2015) used the formulation of Tuncay and Corapcioglu (1997) to study the propagation of Rayleigh waves at the surface of a porous solid saturated with two immiscible viscous fluids. In the present work, a similar formulation is used to study the propagation of cylindrical waves in a no-liquid borehole through a poroelastic solid.

The present study considers a borehole of circular cross-section through a porous solid, which is saturated with a liquid-gas mixture. Cylindrical coordinates are used to formulate the model with radial symmetry about the vertical axis passing through the centre of cylindrical borehole. Frequency equation is derived for the dispersive propagation of surface waves along the curved walls 
of borehole in porous solid formation. A numerical example shows that these cylindrical waves may not be the true surface waves. Even if a true surface wave could propagate, it will be having a very small velocity. A similar situation can also be noticed for the propagation of different modes in Ashish and Tomar (2007). Obviously, such a surface wave may not represent the classical Rayleigh wave, which is observed at the free surface of an elastic half-space. This guides the present study to consider the propagation of pseudo surface wave at the curved surface of the borehole. That means, the velocity of the cylindrical waves along the borehole walls be allowed to increase beyond the velocities of slower dilatational/shear waves in porous surrounding. This yields a complex irrational equation. The complex roots of this complex equation define a general inhomogeneous propagation, which should decay even along the borehole surface as well. But, the target is to find a real phase velocity so that wave motion could decay only in the radial direction. Then, such a real phase velocity needs to satisfy a pair of real equations, resulting from the resolution of the complex dispersion equation. Else, the additional equation could be a restriction to ensure a real phase velocity for pseudo surface wave. This restriction may be solved further to fix the numerical values of the parameters involved in the construction of the mathematical model. In this study, the porous solid refers to composite porous aggregate containing fluids whereas, a drained skeleton is referred as porous frame. Cylindrical waves refer to harmonic waves propagating along the curved surface of the cylindrical borehole and decaying radially into the axially-symmetric walls.

\section{Basic equations}

The three different constituents, i.e., solid grains, pore-gas, pore-liquid, are identified with indices $s, g, l$, respectively. The mixture of these three phases makes a composite porous medium with total connected porosity $f$ and saturation degree $\sigma$ (i.e., the fraction of liquid saturation in connected pore-space). Volume fractions of the constituents are defined as:

$$
\begin{aligned}
& \delta_{s}=1-f, \\
& \delta_{g}=(1-\sigma) f, \\
& \delta_{l}=\sigma f .
\end{aligned}
$$

Following Tuncay and Corapcioglu (1997), constitutive relations for fluid pressures $\left(p^{(g)}, p^{(l)}\right)$ in pores and stresses in porous frame $\left(\tau_{i j}^{(p)}\right)$ are given by

$$
\begin{aligned}
\delta_{s} \tau_{i j}^{(p)}= & \left(a_{11} \nabla \cdot \mathbf{u}+a_{12} \nabla \cdot \mathbf{v}+a_{13} \nabla \cdot \mathbf{w}\right) \delta_{i j} \\
& +G_{p}\left(u_{i, j}+u_{j, i}-\frac{2}{3} u_{k, k} \delta_{i j}\right), \\
-\delta_{g} p^{(g)}= & a_{21} \nabla \cdot \mathbf{u}+a_{22} \nabla \cdot \mathbf{v}+a_{23} \nabla \cdot \mathbf{w}, \\
-\delta_{l} p^{(l)}= & a_{31} \nabla \cdot \mathbf{u}+a_{32} \nabla \cdot \mathbf{v}+a_{33} \nabla \cdot \mathbf{w},
\end{aligned}
$$

where $\delta_{i j}$ is Kronecker symbol. $\mathbf{u}, \mathbf{v}$ and $\mathbf{w}$ denote the displacements of the solid, gas and liquid particles, respectively. Indices (other than $p, g, l$ ) can take values 1, 2 and 3 . The repetition of index $k$ implies summation. $G_{p}$ is the shear modulus for porous frame and the elastic coefficients $a_{i j}$ are as defined in Appendix. Following Lo et al. (2005), the vibrations of constituent particles are considered in an isotropic porous solid, saturated with non-viscous liquid-gas mixture. In the absence of body forces, the equations of motion are given by

$$
\begin{aligned}
\tau_{i j, j}^{(p)} & =\rho_{s} \ddot{u}_{i}, \\
-p_{, i}^{(g)} & =\rho_{g} \ddot{v}_{i}, \\
-p_{, i}^{(l)} & =\rho_{l} \ddot{w}_{i},
\end{aligned}
$$

where $\rho$ 's are intrinsic densities of the constituents. Dot over a variable implies partial derivative with time and comma before an index implies partial space differentiation.

\section{Axi-symmetric motion in a plane}

In cylindrical coordinate system $(r, \theta, z)$, a circular borehole of radius $a$ is considered in a three-phase porous solid of infinite extent. The cylindrical surface $r=a$ makes the porous solid walls of this borehole. Axial symmetry in this medium facilitates to study wave motion in the $r-z$ plane, without losing any information. Hence, all the quantities related to the wave motion become independent of $\theta$-coordinate. Following Sharma (2015), the displacement potentials $\phi_{j},(j=1,2,3,4)$, are used to represent the propagation of four $\left(P_{1}, P_{2}, P_{3}\right.$ and $S V)$ waves in porous solid. Relevant wave equations in the $r-z$ plane are given by 


$$
\begin{aligned}
& \frac{\partial^{2} \phi_{j}}{\partial r^{2}}+\frac{1}{r} \frac{\partial \phi_{j}}{\partial r}+\frac{\partial^{2} \phi_{j}}{\partial z^{2}}=\frac{1}{\alpha_{j}^{2}} \ddot{\phi}_{j}, \quad(j=1,2,3), \\
& \frac{\partial^{2} \phi_{4}}{\partial r^{2}}+\frac{1}{r} \frac{\partial \phi_{4}}{\partial r}-\frac{\phi_{4}}{r^{2}}+\frac{\partial^{2} \phi_{4}}{\partial z^{2}}=\frac{1}{\beta^{2}} \ddot{\phi}_{4}
\end{aligned}
$$

where the velocities $\alpha_{1}, \alpha_{2}, \alpha_{3}$ and $\beta$ are as explained in Appendix. For the propagation of time harmonic waves along $z$-direction, the displacement potentials are given by

$$
\begin{aligned}
\phi_{j}(r, z) & =S_{j} K_{0}\left(k r q_{j}\right) e^{\imath k(z-c t)}, \\
q_{j} & =\sqrt{1-c^{2} / \alpha_{j}^{2}}, \quad(j=1,2,3), \\
\phi_{4}(r, z) & =S_{4} K_{1}\left(k r q_{4}\right) e^{\imath k(z-c t)}, \\
q_{4} & =\sqrt{1-c^{2} / \beta^{2}}
\end{aligned}
$$

where $K_{0}\left(K_{1}\right)$ denotes modified Bessel function of second kind and order 0 (1). The apparent phase velocity $c$ and wave number $k$ define the circular frequency $\omega=k c$. The displacements of constituent particles in the $r-z$ plane are given by

$$
\begin{aligned}
u_{r} & =\sum_{j=1}^{3} \frac{\partial \phi_{j}}{\partial r}-\frac{\partial \phi_{4}}{\partial z}, \\
v_{r} & =\sum_{j=1}^{3} \mu_{j} \frac{\partial \phi_{j}}{\partial r} \\
w_{r} & =\sum_{j=1}^{3} \nu_{j} \frac{\partial \phi_{j}}{\partial r}, \\
u_{z} & =\sum_{j=1}^{3} \frac{\partial \phi_{j}}{\partial z}+\frac{1}{r} \frac{\partial\left(r \phi_{4}\right)}{\partial r}, \\
v_{z} & =\sum_{j=1}^{3} \mu_{j} \frac{\partial \phi_{j}}{\partial z}, \\
w_{z} & =\sum_{j=1}^{3} \nu_{j} \frac{\partial \phi_{j}}{\partial z},
\end{aligned}
$$

where $\mu_{j}$ and $\nu_{j}$ are explained in Appendix. The above expressions are used in (2-4) to calculate the relevant stresses is solid and the fluid-pressures.

\section{Boundary conditions}

The curved walls of an empty borehole is considered to be free of stress, which implies the vanishing of aggregate radial stress as well as tangential stress at the boundary of porous solid. Boreholes are, generally, dug to collect the oils from the porous host. In majority of wells for hydrocarbon exploration, drilling mud is used to maintain the pressure-balance across the borehole boundary. This becomes a necessity to avoid the collapse of borehole when curved wall rocks are not hard enough. But, sometimes it happens that despite the presence of oil in host porous rocks, a dug borehole could provide only gas. Reason may be the size of pores or the permeability of rocks unable to transmit the liquids. The boundary conditions in this study are designed to represent such a likely situation. Hence, a boundary condition is needed to ensure that there is no restriction on the interstitial gas to enter the borehole. Following Dunn (1986), this condition is referred to as the open-pore condition. In the open-pore condition, the pores on the curved surface of the cylinder are exposed to air. Further, to get a no-liquid borehole, a restriction is needed to stop discharge of the pore-liquid out of the surface pores in curved walls of the borehole. This defines an impervious boundary, which is effected through the absence of liquid-pressure gradient in boundary pores (Pride and Berryman 2003). Hence, for the wave propagation in double porosity solid with no-liquid (all gas) borehole, the appropriate boundary conditions at $r=a$ are given by

$$
\begin{aligned}
& \text { (i) } \delta_{s} \tau_{r r}^{(p)}-\delta_{g} p^{(g)}-\delta_{l} p^{(l)}=0, \\
& \text { (ii) } \tau_{z r}^{(p)}=0, \\
& \text { (iii) } p^{(g)}=0, \\
& \text { (iv) } \partial p^{(l)} / \partial r=0 .
\end{aligned}
$$

The last condition ensures the absence of poreliquid discharge out of the surface pores at $r=a$. The above boundary conditions translate into a linear system of four homogeneous equations. Nontrivial solution of this system is ensured through the following equation.

$$
\begin{aligned}
& (2-h)\left(R_{1} q_{1} \eta_{2} \eta_{3}+R_{2} q_{2} \eta_{3} \eta_{1}+R_{3} q_{3} \eta_{1} \eta_{2}\right) \\
& =\left(2 q_{4} \eta_{4}+\frac{h}{k a}\right)\left(Q_{1} \eta_{1} q_{2} q_{3}+Q_{2} \eta_{2} q_{3} q_{1}\right. \\
& \left.\quad+Q_{3} \eta_{3} q_{1} q_{2}\right)
\end{aligned}
$$

where $h=c^{2} / \beta^{2}$ and $\eta_{r}=K_{0}\left(k a q_{r}\right) / K_{1}\left(k a q_{r}\right)$, $(r=1,2,3,4)$. Other coefficients in equation (13) are $R_{i}=Z_{i}\left[\left(2-T_{j} h\right) Y_{l}-\left(2-T_{l} h\right) Y_{j}\right], \quad Q_{i}=$ $2 Y_{i}\left(Z_{j}-Z_{l}\right)$ for cyclic $i, j, l(=1,2,3)$ and with $\epsilon_{j}=\left(\beta / \alpha_{j}\right)^{2}, \quad T_{j}=Z_{j}+\epsilon_{j}\left(a_{11}+\frac{4}{3} G_{p}+a_{12} \mu_{j}+\right.$ 
$\left.a_{13} \nu_{j}\right) / G_{p}, \quad Y_{j}=\epsilon_{j}\left(a_{12}+a_{22} \mu_{j}+a_{32} \nu_{j}\right) / G_{p}, Z_{j}=$ $\epsilon_{j}\left(a_{13}+a_{23} \mu_{j}+a_{33} \nu_{j}\right) / G_{p}$, for $j=1,2,3$.

\section{Surface waves}

Equation (13) is the dispersion equation for propagation of cylindrical waves along the walls of a no-liquid borehole of radius $a$ in a double porosity solid. For non-dimensional wave number $k a \rightarrow \infty$, we have $\eta_{r}=1,(r=1,2,3,4)$, and the equation (13) reduces to

$$
\begin{aligned}
& (2-h)\left(R_{1} q_{1}+R_{2} q_{2}+R_{3} q_{3}\right) \\
& \quad=2 q_{4}\left(Q_{1} q_{2} q_{3}+Q_{2} q_{3} q_{1}+Q_{3} q_{1} q_{2}\right) .
\end{aligned}
$$

This is the secular equation for propagation of Rayleigh wave at the impervious plane boundary of the double porosity solid (Sharma 2015). With the substitution of $Y_{1}=Y_{2}=0$, the equation (13) reduces to

$$
\begin{aligned}
& (2-h)\left[\left(2-T_{1} h\right) \eta_{1} Z_{2} q_{2}-\left(2-T_{2} h\right) \eta_{2} Z_{1} q_{1}\right] \\
& \quad+2\left(2 q_{4} \eta_{4}+\frac{h}{k a}\right)\left(Z_{1}-Z_{2}\right) q_{1} q_{2}=0
\end{aligned}
$$

This is the frequency equation for propagation of cylindrical waves along the curved surface of noliquid borehole in a liquid-saturated porous solid (Sharma and Gogna 1990). These restrictions are chosen to avoid the boundary condition (iii) in equation (12) and the contribution of $P_{3}$ wave to the propagating surface wave. Else, these restrictions yield relations among the elastic coefficients $a_{i j}$. These relations are not so simple that could be solved for well-defined unique physical interpretation. Further, for $k a \rightarrow \infty$, equation (14) is reduced to

$$
\begin{aligned}
& (2-h)\left[\left(2-T_{1} h\right) Z_{2} q_{2}-\left(2-T_{2} h\right) Z_{1} q_{1}\right] \\
& \quad+4 q_{4}\left(Z_{1}-Z_{2}\right) q_{1} q_{2}=0
\end{aligned}
$$

which represents the propagation of Rayleigh waves at the impervious plane boundary of a liquidsaturated porous solid (Deresiewicz 1962). That means, the limit $k a \rightarrow \infty$ is used for $a \rightarrow \infty$, so that the curved surface of borehole behaves as a plane surface and the cylindrical wave becomes analogous to the Rayleigh wave. Else, the limit $k a \rightarrow \infty$, for finite $a$, may represent the high frequency propagation of surface waves along the walls of cylindrical borehole.
For $Y_{1}=Z_{1}=0$, we get $T_{1}=1$ and equation (13) is decomposed into two factors. One of the factors yields

$$
(2-h)^{2} \eta_{1}=2 q_{1}\left(2 q_{4} \eta_{4}+\frac{h}{k a}\right)=0,
$$

which governs the propagation of cylindrical waves along the empty borehole in elastic solid (Biot 1952). In other words, apart from the changes in wave velocities, the contribution of saturated pores to cylindrical waves in a borehole may be represented through the non-zero values of $Y_{1}$ and $Z_{1}$. Further, for $k a \rightarrow \infty$, equation (17) reduces to the secular equation for the propagation of classical Rayleigh wave, i.e., $(2-h)^{2}=4 q_{1} q_{4}$.

\subsection{True surface wave}

True surface waves along the curved surface of porous solid should propagate along the axis of the cylindrical borehole, but decay radially into the porous continuum. Such a wave in porous solid should travel with velocity smaller than all the four body waves in the medium. This implies that the true surface wave will be slower than the slowest dilatational (i.e., $P_{3}$ ) wave also. For the numerical example considered in this study, the velocity of $P_{3}$ wave varies with saturation degree $(\sigma)$, as shown in figure 1 . This velocity increases with capillary parameter $(\chi)$ and reaches up to $185 \mathrm{~m} / \mathrm{s}$ for $\chi=1000$, but it may not be compatible to the velocity of $\mathrm{S}$ wave $(\beta \approx 2.5 \mathrm{~km} / \mathrm{s})$. Note that the velocity $\beta=\sqrt{G_{p} / \delta_{s} \rho_{s}}$ does not change with $\sigma$ or $\chi$. Then, the true surface wave demands that the $c / \beta$ should be $<0.075$. That means, such a slow wave may not be easy to observe in real materials. Further, it undermines the existence of classical Rayleigh wave in real materials, which should propagate at nearly 0.92 times the speed of shear wave. Moreover, for the porous solid considered in this numerical example, it is explored that a true surface wave does not exist for any genuine values of saturation $(\sigma)$, porosity $(f)$ or capillary parameter $(\chi)$.

\subsection{Leaky surface wave}

One option to address the concern in the previous section could be the deviation from true surface wave. That means, the velocity of cylindrical wave at the porous walls be allowed to increase beyond the velocities of slower dilatational waves in the medium, i.e., $c>\alpha_{2}\left(>\alpha_{3}\right)$. Then, for purely 

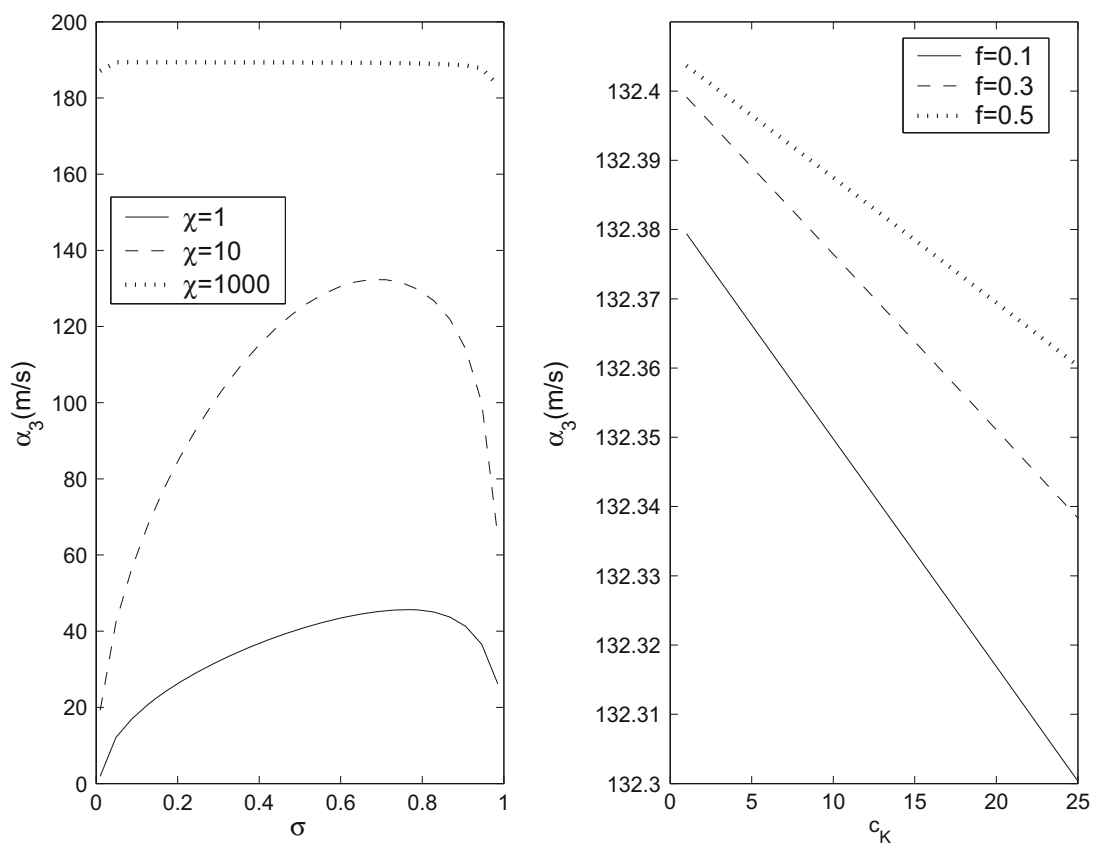

Figure 1. Variations in the velocity of slowest dilatational (i.e., $P_{3}$ ) wave in water $+\mathrm{CO}_{2}$-saturated sandstone with porosity $(f)$, saturation degree $(\sigma)$, capillary pressure $(\chi)$ and consolidation $\left(c_{K} ; c_{G}=1.5 c_{K}\right)$.

imaginary values of $q_{2}$ and $q_{3}$, the secular equation (13) will be a complex irrational equation. Generally, such an equation may not be expected to deliver a real positive solution for $h$. Then, with real $\beta$, a complex $h$ implies a complex $c$, which contradicts the assumption of $c>\alpha_{2}$. On the other hand, a complex $c$ represents a leaky cylindrical wave, which decays while propagating in the interior of the medium or along the boundary. But, with an elastic frame and inviscid pore-fluids, the porous solid should behave non-dissipative to the wave propagation. Such a medium demands that any wave propagating in the interior or at the surface should have a real velocity.

\subsection{Pseudo surface wave}

The demand of real phase velocity $c$ in nondissipative porous medium motivates to hunt for a real solution of equation (13). That means, to consider a pseudo surface wave along the borehole walls, which propagates with real phase velocity $c>\alpha_{2}$. Hence, for $h \in\left(\epsilon_{2}^{-1}, 1\right)$, this defines real $s_{j}=\sqrt{h \epsilon_{j}-1},(j=2,3)$. Then, with $q_{2}=\imath s_{2}$ and $q_{3}=\imath s_{3}$, the complex equation (13) is resolved into two real equations as follows.

$$
(2-h) X_{1}+\left(2 q_{4} \eta_{4}+\frac{h}{k a}\right) X_{2}=0,
$$

$$
\begin{aligned}
(2-h) X_{3}-\left(2 q_{4} \eta_{4}+\frac{h}{k a}\right) X_{4}=0 \\
X_{1}=R_{1} q_{1}\left(\eta_{2 R} \eta_{3 R}-\eta_{2 I} \eta_{3 I}\right)-R_{2} s_{2} \eta_{1} \eta_{3 I} \\
\quad-R_{3} s_{3} \eta_{1} \eta_{2 I} \\
X_{2}=Q_{1} \eta_{1} s_{2} s_{3}+Q_{2} q_{1} \eta_{2 I} s_{3}+Q_{3} q_{1} \eta_{3 I} s_{2} \\
X_{3}=R_{1} q_{1}\left(\eta_{2 R} \eta_{3 I}+\eta_{2 I} \eta_{3 R}\right)+R_{2} s_{2} \eta_{1} \eta_{3 R} \\
\quad+R_{3} s_{3} \eta_{1} \eta_{2 R} \\
X_{4}=q_{1}\left(Q_{2} \eta_{2 R} s_{3}+Q_{3} \eta_{3 R} s_{2}\right)
\end{aligned}
$$

where $\eta_{j R}\left(\eta_{j I}\right)$ denotes the real (imaginary) part of $\eta_{j}$. For a fixed value of non-dimensional wave number $k a$, these two equations involve $h$ as only unknown. The condition for a real value of $h$ to satisfy these two equations is given by

$$
X_{1} X_{4}+X_{2} X_{3}=0 \text {. }
$$

This condition may be solved to restrict one or more parameters, which are used in the mathematical model for partially-saturated porous solid. Then, subject to such restrictions, the dispersion of phase velocity (or $h$ ) is calculated from

$$
(2-h) X_{1}+\left(2 q_{4} \eta_{4}+\frac{h}{k a}\right) X_{2}=0 .
$$

In other words, an ill-defined system (equation 18) provides an opportunity to estimate/verify the parametric values of various physical properties 
of the medium (i.e., porosity, saturation degree, capillary-pressure, consolidation, etc.), so as to ensure the propagation of surface waves.

Note that the restriction $Z_{1}=Z_{2}=Z_{3}$ ensures that $Q_{1}=Q_{2}=Q_{3}=0$. Then, we have $X_{2}=$ $X_{4}=0$, which satisfies the requirement for real $h$ in equation (19). But, this reduces equation (20) to $(2-h) X_{1}=0$, which implies the propagation of pseudo surface wave with phase velocity $c=\sqrt{2} \beta$. Else, the propagation of cylindrical waves is governed by the dispersion equation, given by

$$
\begin{aligned}
& R_{1} q_{1}\left(\eta_{2 R} \eta_{3 R}-\eta_{2 I} \eta_{3 I}\right)-R_{2} s_{2} \eta_{1} \eta_{3 I} \\
& \quad-R_{3} s_{3} \eta_{1} \eta_{2 I}=0 .
\end{aligned}
$$

The restriction $Z_{1}=Z_{2}=Z_{3}$ is solved further into a system of two equations given by

$$
\begin{aligned}
& a_{13}+a_{23} \mu_{12}+a_{33} \nu_{12}=0 \\
& a_{13}+a_{23} \mu_{23}+a_{33} \nu_{23}=0
\end{aligned}
$$

with $\mu_{i j}=\left(\mu_{i} \alpha_{j}^{2}-\mu_{j} \alpha_{i}^{2}\right) /\left(\alpha_{j}^{2}-\alpha_{i}^{2}\right)$ and $\nu_{i j}=$ $\left(\nu_{i} \alpha_{j}^{2}-\nu_{j} \alpha_{i}^{2}\right) /\left(\alpha_{j}^{2}-\alpha_{i}^{2}\right)$.

In another type of pseudo surface wave in borehole, the phase velocity is allowed to increase beyond the velocity of shear wave as well. That means, $c>\beta\left(>\alpha_{2}>\alpha_{3}\right)$, which yields imaginary $q_{4}=\imath s_{4}$ with real $s_{4}=\sqrt{h-1}$ and complex $\eta_{4}=\eta_{4 R}+\imath \eta_{4 I}$. Then, the condition (19) for existence of real $h \in\left(1, \epsilon_{1}^{-1}\right)$ changes to

$$
\begin{aligned}
& X_{1}^{\prime} X_{4}^{\prime}+X_{2}^{\prime} X_{3}^{\prime}=0 ; \\
& X_{1}^{\prime}=X_{1}+s_{4}\left(\eta_{4 R} X_{4}-\eta_{4 I} X_{2}\right), \\
& X_{2}^{\prime}=X_{2}-k a X_{1}, \\
& X_{3}^{\prime}=X_{3}+s_{4}\left(\eta_{4 R} X_{2}+\eta_{4 I} X_{4}\right), \\
& X_{4}^{\prime}=X_{4}+k a X_{3} .
\end{aligned}
$$

\section{Numerical example}

A particular numerical example is designed to illustrate the results of the present study. A reservoir rock (Berea sandstone) with water and $\mathrm{CO}_{2}$ bubbles in its pores makes the physical model of three phase porous medium. The solid grains with elastic moduli $K_{s}=38 \mathrm{GPa}, G_{s}=44 \mathrm{GPa}$ and density $\rho_{s}=2650 \mathrm{~kg} / \mathrm{m}^{3}$ form the skeletal frame of porosity $f=0.3$. Then, the elastic moduli for porous frame $\Gamma_{p}=(1-f) \Gamma_{s} /\left(1+f c_{\Gamma}\right), \Gamma=K, G$ are defined through two consolidation parameters $c_{K}$ and $c_{G}$. A mixture of $\mathrm{CO}_{2}$ bubbles (bulk modulus $K_{g}=3.7 \mathrm{MPa}$, density $\rho_{g}=103 \mathrm{~kg} / \mathrm{m}^{3}$ ) in water (bulk modulus $K_{l}=2.25 \mathrm{GPa}$, density $\left.\rho_{l}=1000 \mathrm{~kg} / \mathrm{m}^{3}\right)$ is filling the pore space. For partial saturation (of degree $\sigma$ ), the capillary effect is accounted through the equivalent bulk modulus $K_{c}=\chi \sigma(1-\sigma) / K_{g}$. The parameter $\chi$ is specified through a choice of some fitting constants on inertial couplings (Lo et al. 2005).

The numerical values of various parameters are used to calculate the velocities of the four waves in porous solid. Figure 1 exhibits the effect of capillary $(\chi)$, saturation $(\sigma)$, porosity $(f)$ and consolidation $\left(c_{K} ; c_{G}=1.5 c_{K}\right)$ on the velocity of $P_{3}$ wave. Note that this velocity is important in studying the existence of true surface waves in partially saturated porous solid. The right plot in this figure clearly shows that porosity and consolidation have almost no effect on the velocity $\left(\alpha_{3}\right)$ of the slowest wave in porous solid. The left plot in this figure shows the increase of velocity with the increase of capillary parameter $(\chi)$. The effect of saturation on $\alpha_{3}$ is also quite significant, except for very high value of $\chi$. Note that each of the frequency equation from (13) to (18) is solved for the unknown $h$, which involves the velocity $\beta$ as $h=c^{2} / \beta^{2}$. This velocity of shear wave does not depend on the capillary parameter $(\chi)$ or the saturation degree $(\sigma)$. The effect of porosity $(f)$ and consolidation $\left(c_{K} ; c_{G}=1.5 c_{K}\right)$ on $\beta$ is exhibited in figure 2 . Clearly, the velocity of shear wave decreases with the increase of porosity as well as the consolidation parameter $c_{G}$.

It is noted that the relation $k=\omega / c$ inducts the involvement of phase velocity $c$ in non-dimensional

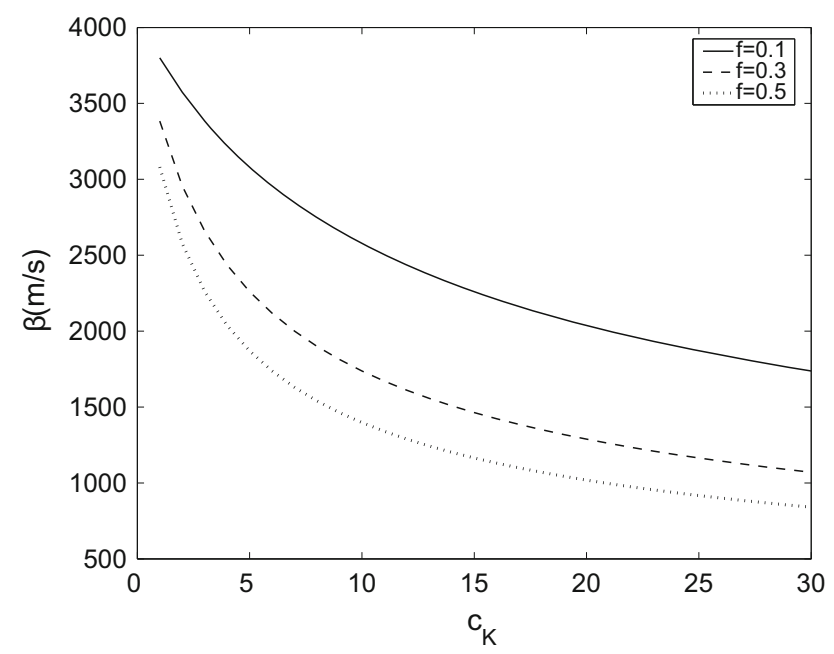

Figure 2. Variations in the velocity of shear wave in water $+\mathrm{CO}_{2}$-saturated sandstone with porosity $(f)$ and consolidation $\left(c_{K}, c_{G}=1.5 c_{K} ; \sigma=0.7, \chi=10\right)$. 


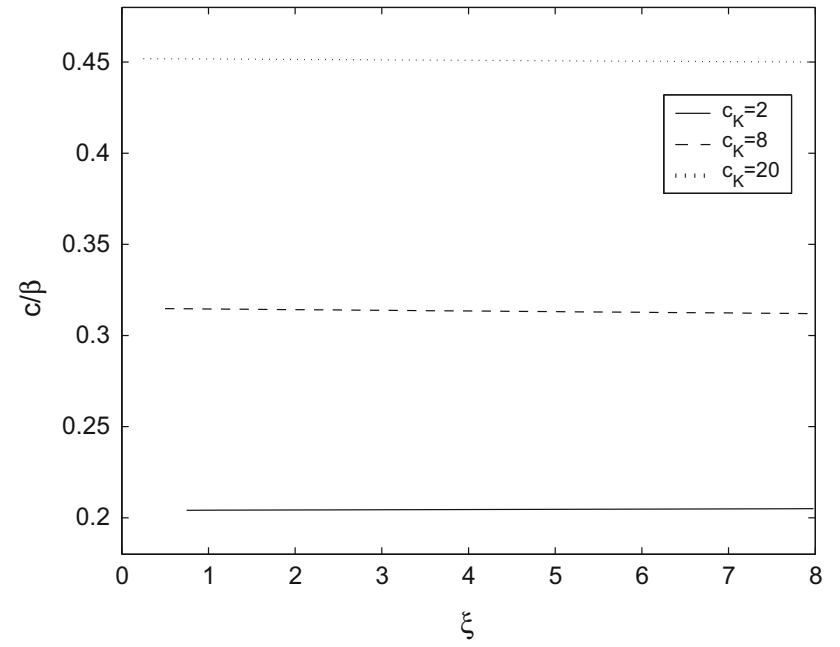

Figure 3. Dispersion of phase velocity of cylindrical wave in borehole through water-saturated sandstone $(f=0.3, \sigma=$ $\left.0.7, \chi=10, c_{G}=1.5 c_{K}\right)$.

wave number $k a$. Then, a relation $k a=(\omega a / \beta) /$ $\sqrt{h}=\xi \sqrt{1+c_{G} f} / \sqrt{h}$ defines a non-dimensional frequency $\xi=\left(\omega a / \sqrt{G_{s} / \rho_{s}}\right)$, which is independent of porosity $(f)$, saturation degree $(\sigma)$, capillary $(\chi)$, consolidation $\left(c_{K}, c_{G}\right)$ and phase velocity. Hence, the frequency $\xi$ is preferred to define the dispersion in phase velocity $c$ (or $c / \beta$ ) of cylindrical waves. Equation (13) is solved numerically for $h$ with given $\xi$ and the genuine variations of $f, \sigma, \chi, c_{K}$. This equation does not yield a real $h<\epsilon_{3}^{-1}$, which defines the existence of true surface wave. This implies that true surface wave does not propagate along the borehole walls in partially-saturated porous solid. However, such a wave does propagate when the medium supporting the borehole is an elastic solid or fully-saturated porous solid. That means, equation (15) is satisfied for some $h\left(<\epsilon_{2}^{-1}\right)$ and some $h(<1)$ satisfies equation (17). However, in each case, the non-dimensional frequency $\xi$ (or $k a$ ) must be above some minimum value.

In figure 3, dispersion is shown for cylindrical waves in empty borehole across the fully-saturated porous solid. Note that the cylindrical wave does not propagate for $\xi \ll 1$. However, this restriction relaxes with the increase of consolidation parameter $c_{K}$ (and hence $c_{G}=1.5 c_{K}$ ). This implies that in a highly consolidated material (i.e., elastic solid), true surface wave can propagate in borehole at any frequency. Dispersion (decrease of $c / \beta$ with $\xi$ ) is very small, but improves a bit with the increase of $c_{K}$. Note that with the increase of $c_{K}, \beta$ decreases (figure 2 ), but $c / \beta$ increases. This ensures a much faster propagation of cylindrical waves in a

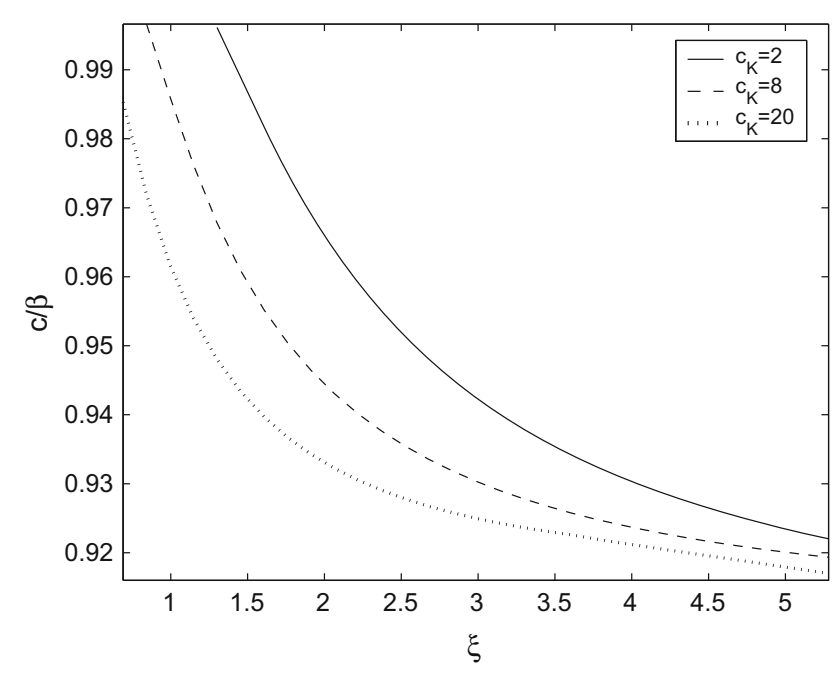

Figure 4. Dispersion of phase velocity of cylindrical wave in borehole through dry/drained sandstone $\left(f=0.3, c_{G}=\right.$ $\left.1.5 c_{K}\right)$.

porous solid with higher consolidation parameters. Figure 4 exhibits the propagation of cylindrical waves in dry sandstone, which is equivalent to an ordinary elastic solid. Again the restriction applies on the value of $\xi$, which relaxes with the decrease (increase) of elastic moduli (consolidation parameters) and $\beta$. With the increase of $\xi$, the phase velocity of cylindrical waves decreases from $\beta$ (i.e., $c / \beta \approx 1)$ to the velocity of Rayleigh wave in elastic solid (i.e., $c / \beta \approx 0.92$ ). In fact, Rayleigh wave in dry porous solid should propagate with $c / \beta$ around 0.92 , as shown in figure 4 . Whereas, in case of fully saturated porous solid, the slowest wave is $P_{2}$ wave with velocity $\left(\alpha_{2}\right)$ much smaller than the velocity $(\beta)$ of the shear wave. Now, the true surface wave must be slower than this slowest wave. Then, obviously for $c / \alpha_{2}<1$, the value of $c / \beta$ will be much lesser than $c / \alpha_{2}$. This being the reason that the phase speed of surface wave $(c / \beta)$ drops down significantly in figure 3 in contrast to the higher phase speed in figure 4 . In addition to this, the slow moving surface wave in fully saturated sandstone is not as dispersive as the faster one in dry host.

\subsection{Pseudo surface wave}

As explained in the previous section, the restriction $Z_{1}=Z_{2}=Z_{3}$ translates into vanishing of two expressions in equation (22). These expressions are computed for varying values of parameters for porosity $(f)$, saturation $(\sigma)$, consolidation $\left(c_{K} ; c_{G}=1.5 c_{K}\right)$ and capillary $(\chi)$. From the plots in figure 5 , it is observed that these expressions may not vanish for the genuine values of these 

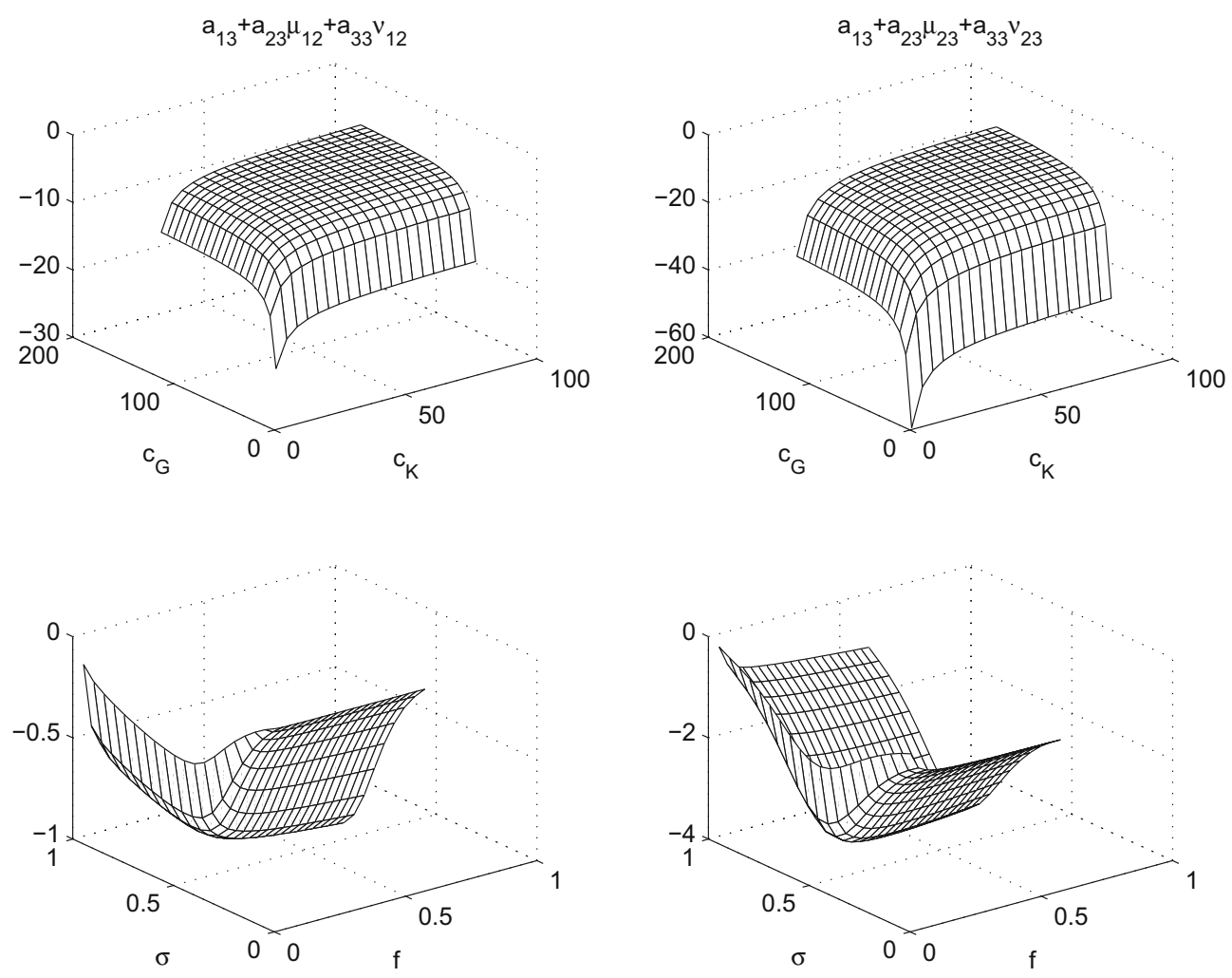

Figure 5. Absence of pseudo surface wave in water $+\mathrm{CO}_{2}$-saturated sandstone when $Z_{1}=Z_{2}=Z_{3}$ \{cf. equation (22): variations in $c_{K}-c_{G}(f=0.3, \sigma=0.7)$ and in $\left.f-\sigma\left(c_{K}=15, c_{G}=1.5 c_{K}\right)\right\}$.

parameters. The possibility of vanishing may be either at $\sigma=1$ or at $\sigma=0$. Recall that $\sigma=1$ represents the liquid-saturated porous solid with single porosity, for which the dispersion of cylindrical waves is displayed in figure 3 . The case of $\sigma=0$ yields $a_{13}=a_{23}=a_{33}=0$ (from Appendix), which satisfies the system equation (22) without any restriction. Then, the dispersion equation (21) should govern the propagation of cylindrical waves through borehole in dry/drained porous solid. Surprisingly, as shown in figure 6 , this equation yields $c=\sqrt{2} \beta$ (i.e., $h=2$ ) for any value of $f, c_{K}, c_{G}$ or non-dimensional frequency $\xi$. This implies the propagation of non-dispersive cylindrical wave with fixed phase velocity $c=\sqrt{2} \beta \in\left(\beta, \alpha_{1}\right)$.

To check any possibility of pseudo surface wave with velocity $c \in\left(\alpha_{2}, \beta\right)$, the expression $X_{1} X_{4}+$ $X_{2} X_{3}$ is computed for varying values of $\left(f, \sigma, c_{K}\right.$, $\chi, \xi)$. From the two rows of plots in figure 7 , the corresponding plots certify that sign of $X_{1} X_{4}+$ $X_{2} X_{3}$ does not change for $c \in\left(\alpha_{2}, \beta\right)$. This implies that any cylindrical wave in considered sandstone cannot have the velocity between $\alpha_{2}$ and $\beta$. Then, there remains a possibility of pseudo surface wave with phase velocity $c \in\left(\beta, \alpha_{1}\right)$. As discussed in the previous section, the existence of such a wave

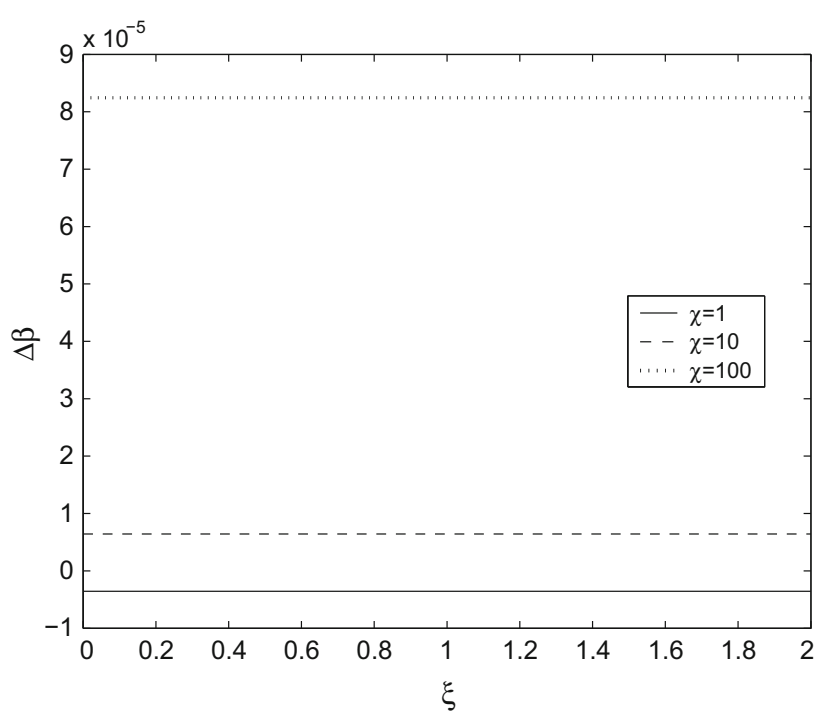

Figure 6. Phase velocity of pseudo surface wave in borehole through sandstone filled only with $\mathrm{CO}_{2}(\sigma=0, f=$ $\left.0.3, c_{K}=15, c_{G}=1.5 c_{K}\right)$; deviation of $c / \beta$ from $\sqrt{2}$ $(\Delta \beta=c / \beta-\sqrt{2})$.

is governed by the relation (23). So, the expression $X_{1}^{\prime} X_{4}^{\prime}+X_{2}^{\prime} X_{3}^{\prime}$ is computed for $h=1.01$ (i.e., $c>\beta$ ) and $h=\epsilon_{1}^{-1}-0.01$ (i.e., $\left.c<\alpha_{1}\right)$. The variations of this expression with $f, \sigma, c_{K}, \chi$ and $\xi$ are displayed 

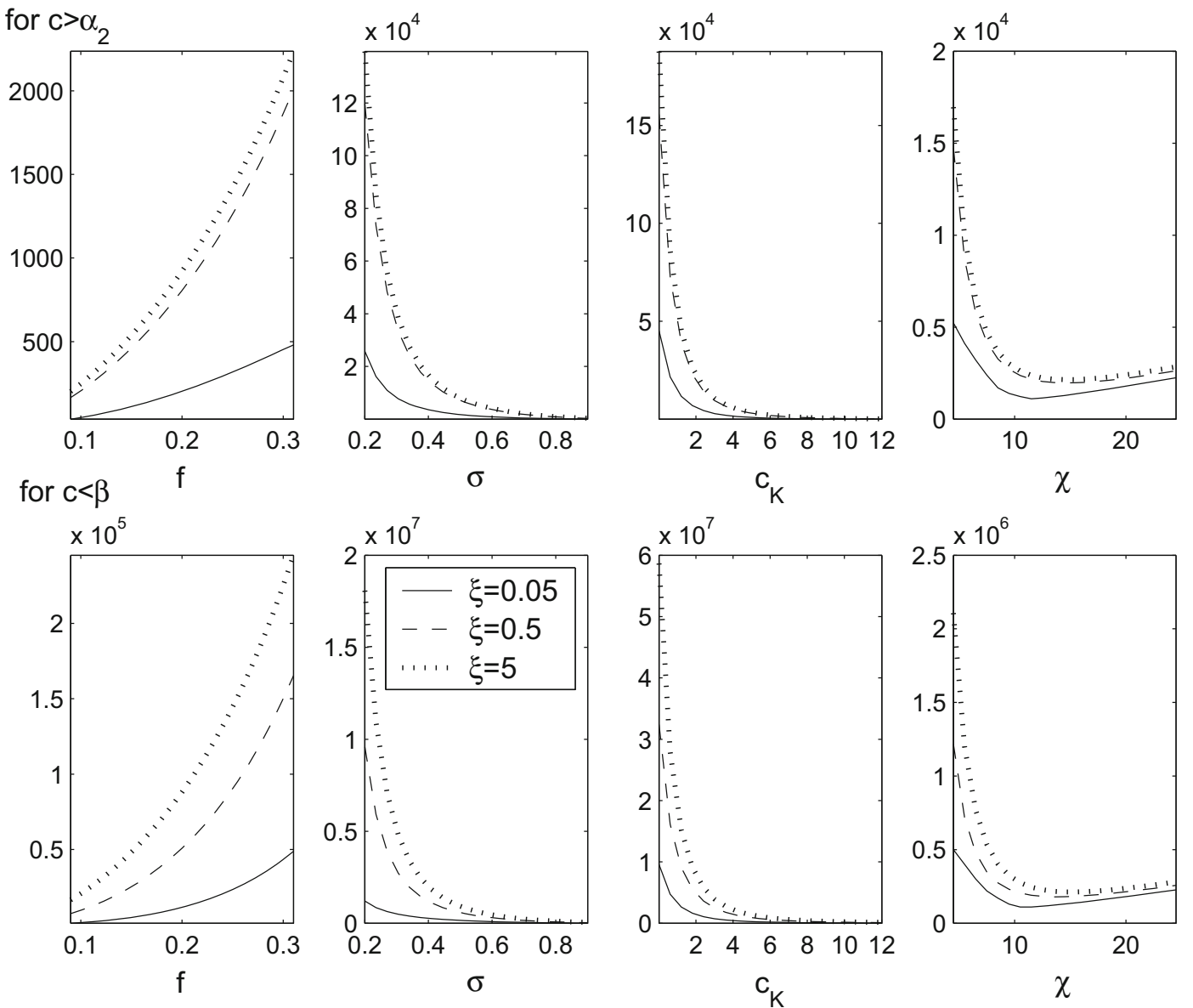

Figure 7. Absence of pseudo surface wave in water $+\mathrm{CO}_{2}$-saturated sandstone for $c \in\left(\alpha_{2}, \beta\right)$; $\{$ cf. equation (19): variations in $f, \sigma, c_{K}\left(c_{G}=1.5 c_{K}\right)$ and $\left.\chi\right\}$.

in figure 8. On comparing the corresponding plots of two rows in this figure, the change of sign clearly indicates the existence of such a cylindrical wave for $\xi=2,5$ but not for $\xi=0.5$.

\section{Concluding remarks}

The present study explores the propagation of cylindrical waves in a no-liquid cylindrical borehole through partially-saturated porous solid. Such a wave is expected to be a true surface wave, which propagates along the curved walls of the borehole and decays into the solid continuum. With pores saturated by twin-fluid mixture, particularly air restricting the complete saturation by liquid, this study considers a realistic model to study real-time changes in reservoir rocks around active/deserted wells. The variable liquid share in pores enables to represent the pore saturation from almost dry to fully-saturated. Flexibility and versatility of the mathematical model are demonstrated by deducing the frequency equations for cylindrical/
Rayleigh waves in single porosity medium or ordinary elastic medium. Numerical results are obtained for a particular model and may not qualify for generalisation. But the qualitative interpretation of these results may be useful in exploration seismology. Some of such results are explained as follows.

- Cylindrical wave in a borehole may be the true surface wave when the medium supporting the borehole is an elastic solid or fully-saturated porous solid. Such a wave cannot be the true surface wave when borehole lies in a partiallysaturated porous solid.

- A true surface wave in a borehole can propagate only at frequencies above some minimum value. A similar restriction is found for the propagation of surface waves in borehole through elastic solid (Biot 1952) or liquid-saturated porous solid (Sharma and Gogna 1990). This lower frequency limit depends on the radius of the borehole as well as the geophysical properties of the supporting medium. For example, with the numerical 

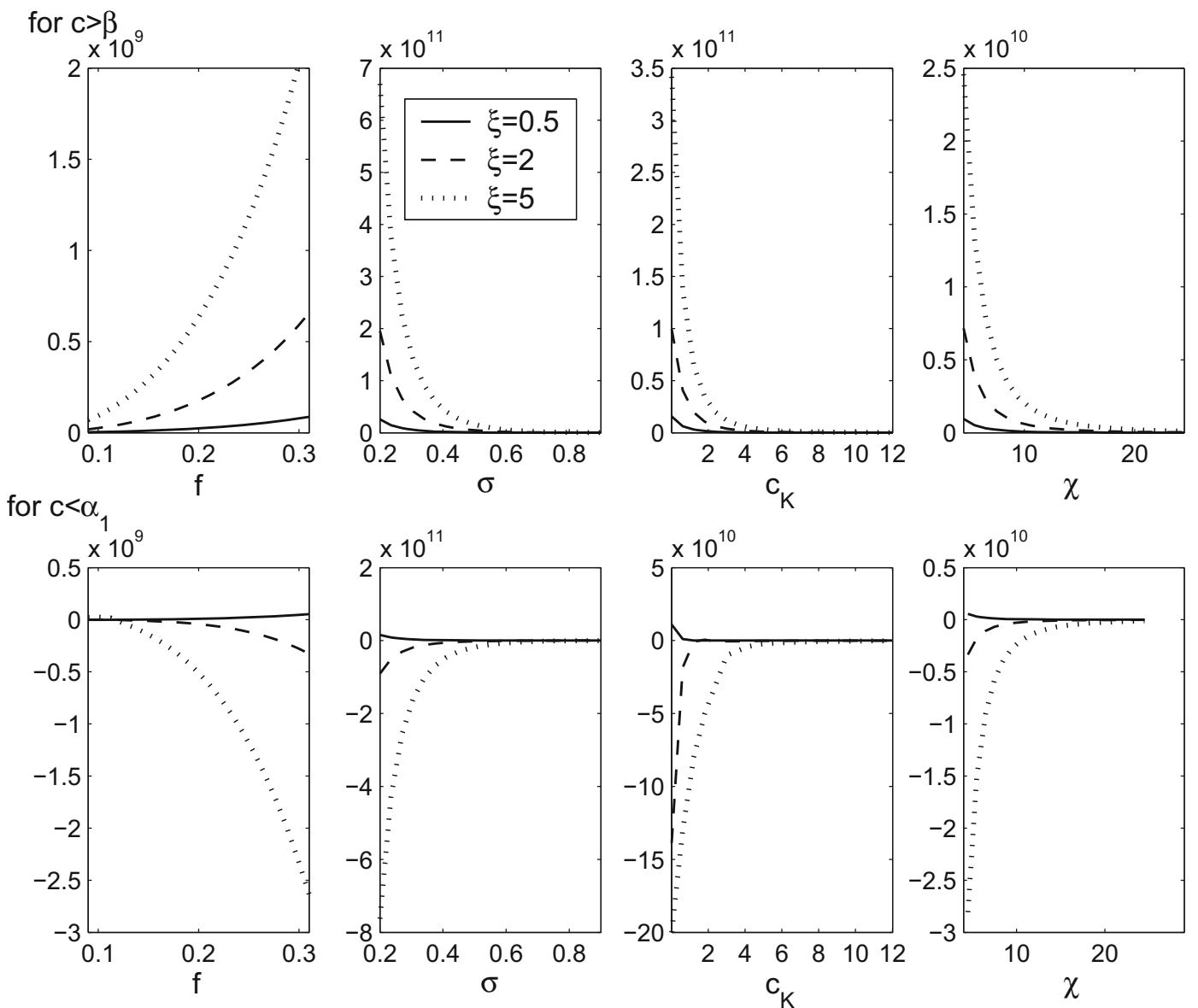

Figure 8. Existence of pseudo surface wave in water $+\mathrm{CO}_{2}$-saturated sandstone for $c \in\left(\beta, \alpha_{1}\right)$; $\{$ cf. equation (23): variations in $f, \sigma, c_{K}\left(c_{G}=1.5 c_{K}\right)$ and $\left.\chi\right\}$.

values considered in the present study and $c_{K}=$ 8 , Rayleigh wave may not propagate for $\omega a<$ $700 \mathrm{~m} / \mathrm{s}$, i.e., a limiting frequency of $100 \mathrm{~Hz}$ for a borehole of radius one meter. However, this limit decreases further with the increase of consolidation parameters.

- Cylindrical wave in a borehole can be the pseudo surface wave. In dry/drained porous solid, such a wave can propagate at a fixed velocity of $\sqrt{2}$ times the speed of shear wave in the medium. This velocity does not alter with the changes in elastic moduli, porosity, frequency or radius of borehole.

- Phase velocity of the pseudo surface waves in borehole through partially-saturated porous solid exceeds the velocity of shear wave. That means, such a leaky wave should propagate faster than shear wave but slower than the fastest dilatational wave in porous surrounding.

Pseudo (or leaky) surface acoustic waves (PSAW) can be experimentally observed on numerous crystals using surface Brillouin scattering and acoustic microscopy (Aleksandrov et al. 1992). Application of PSAW are found in the evaluation of phononic crystals (Graczykowski et al. 2016) in the design and optimisation of PnCs based high efficiency acoustic filters, reflectors, or waveguides (Briggs 1992). In the dictionary of exploration geophysics, pseudo-Rayleigh waves are identified as the ground roll, which is a particular type of surface wave that travels along or near the ground surface. Ground roll in seismic records is used as a tool to explore the lateral variations of shear-wave velocity (AlEqabi and Herrmann 1993; Socco et al. 2009). Spectral analysis of surface waves (SASW) is used to analyse the ground roll dispersion in relation to produce near-surface S-wave velocity profiles (Nazarian et al. 1983). This method is quite effective for estimating $\mathrm{S}$-wave velocity in near-surface materials (soil, rocks, and pavements), which are of fundamental interest in many groundwater, engineering, and environmental studies (Craig 1992). S-wave velocity profiles are also used in stiffness determinations for construction engineering and seismic response studies. 


\section{Acknowledgement}

Author is grateful to the unknown reviewers for their contribution in improving the manuscript.

\section{Appendix}

In terms of measurable quantities, elastic constants in three-phase porous solid are given by

$$
\begin{aligned}
a_{11} & =K_{p}, \\
a_{12} & =a_{21}=K_{g} \delta_{s}(1-\sigma)\left(K_{c}+K_{l}\right) / K, \\
a_{13} & =a_{31}=K_{l} \delta_{s} \sigma\left(K_{c}+K_{g}\right) / K, \\
a_{22} & =K_{g} \delta_{g}\left[(1-\sigma) K_{l}+K_{c}\right] / K, \\
a_{23} & =a_{32}=K_{g} K_{l}(1-\sigma) \delta_{l} / K, \\
a_{33} & =K_{l} \delta_{l}\left(K_{g} \sigma+K_{c}\right) / K, \\
K & =K_{g} \sigma+K_{c}+K_{l}(1-\sigma), \\
K_{c} & =\chi \sigma(1-\sigma) K_{g}
\end{aligned}
$$

where $K_{p}, K_{g}$ and $K_{l}$ are the bulk moduli of porous frame, gas and liquid phases respectively. The two pore-fluids are assumed immiscible. For miscible pore-fluids, constitutive relations involve Henry's constant (Garg and Nayfeh 1986). As a result, the elastic coefficients $a_{i j}$ in relations $(2-4)$ cease to be symmetric (i.e., $a_{i j} \neq a_{j i}$ ). The saturation degree $\sigma \in(0,1)$ measures the share of liquid in filling the pores. $K_{c}$ is the equivalent bulk modulus (Garg and Nayfeh 1986) for macroscopic capillary pressure between wetting and non-wetting fluids. For partial saturation (i.e., $0<\sigma<1$ ), a capillary parameter $(\chi)$ is used to fix the value of $K_{c}$ relative to the $K_{g}$.

Following Sharma (2012), three dilatational $\left(P_{1}\right.$, $\left.P_{2}, P_{3}\right)$ waves and a shear $(S)$ wave propagate in a three phase non-dissipative porous solid. The velocities $\left(\alpha_{1}>\alpha_{2}>\alpha_{3}\right)$ of three dilatational waves are derived from the roots of a cubic equation in $\alpha^{2}$, given by

$$
D \alpha^{6}-C \alpha^{4}+B \alpha^{2}-A=0 .
$$

Coefficients in this cubic equation are defined as follows.

$$
\begin{gathered}
A=\left(a_{11}+\frac{4}{3} G_{p}\right) A_{1}+a_{12} A_{2}+a_{13} A_{3}, \\
B=\left(a_{11}+\frac{4}{3} G_{p}\right) B_{1}-a_{12} B_{2}-a_{13} B_{3}+\delta_{s} \rho_{s} A_{1}, \\
C=\left(a_{11}+\frac{4}{3} G_{p}\right) C_{1}+\delta_{s} \rho_{s} B_{1}, \quad D=\delta_{s} \rho_{s} C_{1},
\end{gathered}
$$

where,

$$
\begin{aligned}
& A_{1}=a_{22} a_{33}-a_{23} a_{23}, \\
& A_{2}=a_{23} a_{13}-a_{12} a_{33}, \\
& A_{3}=a_{12} a_{23}-a_{13} a_{22}, \\
& B_{1}=a_{22} \delta_{l} \rho_{l}+a_{33} \delta_{g} \rho_{g}, \\
& B_{2}=a_{12} \delta_{l} \rho_{l}, \\
& B_{3}=a_{13} \delta_{g} \rho_{g}, \\
& C_{1}=\delta_{g} \rho_{g} \delta_{l} \rho_{l} .
\end{aligned}
$$

The velocity of lone shear wave in porous solid is given by $\beta=\sqrt{G_{p} / \delta_{s} \rho_{s}}$.

The coefficients for fluid-solid coupling in (1011) are given by

$$
\begin{aligned}
\mu_{j} & =\frac{A_{2}-B_{2} \alpha_{j}^{2}}{A_{1}-B_{1} \alpha_{j}^{2}+C_{1} \alpha_{j}^{4}}, \\
\nu_{j} & =\frac{A_{3}-B_{3} \alpha_{j}^{2}}{A_{1}-B_{1} \alpha_{j}^{2}+C_{1} \alpha_{j}^{4}}, \quad(j=1,2,3) .
\end{aligned}
$$

\section{References}

Aleksandrov V V, Velichkina T S, Mozhaev V G, Potapova Ju B, Khmelev A K and Yakovlev I A 1992 New data concerning surface Mandelstamm-Brillouin light scattering from basal plane of germanium crystal; Phys. Lett. A162 418-422.

Al-Eqabi G I and Herrmann R B 1993 Ground roll: A potential tool for constraining shallow shear-wave structure; Geophysics 58 713-719.

Ashish A and Tomar S K 2007 Elastic waves along a cylindrical borehole in a poroelastic medium saturated by two immiscible fluids; J. Earth Syst. Sci. 116 225-234.

Biot M A 1952 Propagation of elastic waves in a cylindrical bore containing a fluid; J. Appl. Phys. 23 997-1005.

Biot M A 1956 The theory of propagation of elastic waves in a fluid-saturated porous solid. I: Low-frequency range, II: Higher frequency range; J. Acoust. Soc. Am. 28 168-191.

Biot M A 1962a Mechanics of deformation and acoustic propagation in porous media; J. Appl. Phys. 33 1482-1498.

Biot M A 1962b Generalized theory of acoustic propagation in porous dissipative media; J. Acoust. Soc. Am. 341254 1264.

Bowen R M 1976 The Theory of Mixtures; Continuum Physics, Vol. 3 (ed.) A C Eringen, Academic Press, New York.

Briggs A 1992 Acoustic Microscopy; Clarendon, Oxford, Chapter 11.

Brutsaert W 1964 The propagation of elastic waves in unconsolidated unsaturated granular mediums; J. Geophys. Res. $69243-257$.

Chao G, Smeulders D M J and van Dongen M E H 2004 Shock-induced borehole waves in porous formations: Theory and experiments; J. Acoust. Soc. Am. 116 693-702. 
Chao G, Smeulders D M J and van Dongen M E H 2007 Seismic signatures of partial saturation on acoustic borehole modes; Geophysics 72 E77-E86.

Cheng C H, Jinzhong Z and Burns D R 1987 Effects of in situ permeability on the propagation of Stoneley (tube) waves in a borehole; Geophysics 52 1279-1289.

Corapcioglu M Y and Tuncay K 1996 Propagation of Waves in Porous Media; Advances in Porous Media, Vol. 3 (ed.) Corapcioglu M Y, Elsevier, Amsterdam.

Craig R F 1992 Soil Mechanics; 5th edn, Chapman \& Hall, New York.

Deresiewicz H 1962 The effect of boundaries on wave propagation in a liquid-filled porous solid: IV. Surface waves in a half-space; Bull. Seismol. Soc. Am. 52 627-638.

Dunn K-J 1986 Acoustic attenuation in fluid-saturated porous cylinders at low frequencies; J. Acoust. Soc. Am. 79 1709-1721.

Garg S K and Nayfeh A H 1986 Compressional wave propagation in liquid and/or gas saturated elastic porous media; J. Appl. Phys. 60 3045-3055.

Graczykowski B, Alzina F, Gomis-Bresco J and SotomayorTorres C M 2016 Finite element analysis of true and pseudo surface acoustic waves in one-dimensional phononic crystals; J. Appl. Phys. 119 025308, https:// doi.org/10.1063/1.4939825.

Li P and Schanz M 2011 Wave propagation in a 1-D partially saturated poroelastic column; Geophys. J. Int. $1841341-$ 1353.

Lo W-C, Sposito G and Majer E 2005 Wave propagation through elastic porous media containing two immiscible fluids; Water Resour. Res. 41, https://doi.org/10.1029/ 2004WR003162.

Nazarian S, Stokoe I I, Kenneth H and Hudson W R 1983 Use of spectral analysis of surface waves method for determination of moduli and thicknesses of pavement systems; Transp. Res. Rec. 930 38-45.

Pride S R and Berryman J G 2003 Linear dynamics of double-porosity dual-permeability materials, II. Fluid transport equations; Phys. Rev. E 68036604.

Santos J E, Corbero J M and Douglas J 1990a Static and dynamic behaviour of a porous solid saturated by a twophase fluid; J. Acoust. Soc. Am. 87 1428-1438.

Santos J E, Douglas J, Corbero J M and Lovera O M 1990b A model for wave propagation in a porous medium saturated by a two-phase fluid; J. Acoust. Soc. Am. 87 14391448.

Sharma M D 2012 Rayleigh waves in a partially-saturated poroelastic solid; Geophys. J. Int. 189 1203-1214.

Sharma M D 2015 Propagation of Rayleigh waves in a partially-saturated porous solid with impervious boundary; Eur. J. Mech.-A: Solids 49 158-168.

Sharma M D and Gogna M L 1990 Propagation of elastic waves in a cylindrical bore in a liquid saturated porous solids; Geophys. J. Int. 103 47-54.

Socco L V, Boiero D, Foti S and Wisen R 2009 Laterally constrained inversion of ground roll from seismic reflection records; Geophysics $\mathbf{7 4}$ G35-G45.

Tuncay K and Corapcioglu M Y 1997 Wave propagation in poroelastic media saturated by two fluids; J. Appl. Mech. $64313-330$

Corresponding editor: M RADHAKRISHNA 